\title{
Flettner Rotor Concept for Marine Applications: A Systematic Study
}

\author{
A. De Marco, S. Mancini, C. Pensa, G. Calise, and F. De Luca \\ Department of Industrial Engineering, University of Naples Federico II, Via Claudio 21, 80125 Naples, Italy \\ Correspondence should be addressed to S. Mancini; simone.mancini@unina.it
}

Received 19 January 2016; Revised 31 May 2016; Accepted 15 June 2016

Academic Editor: Ryoichi Samuel Amano

Copyright ( 2016 A. De Marco et al. This is an open access article distributed under the Creative Commons Attribution License, which permits unrestricted use, distribution, and reproduction in any medium, provided the original work is properly cited.

\begin{abstract}
The concept of Flettner rotor, a rotating cylinder immersed in a fluid current, with a top-mounted disk, has been analyzed by means of unsteady Reynolds averaged Navier-Stokes simulations, with the aim of creating a suitable tool for the preliminary design of the Flettner rotor as a ship's auxiliary propulsion system. The simulation has been executed to evaluate the performance sensitivity of the Flettner rotor with respect to systematic variations of several parameters, that is, the spin ratio, the rotor aspect ratio, the effect of the end plates, and their dimensions. The Flettner rotor device has been characterized in terms of lift and drag coefficients, and these data were compared with experimental trends available in literature. A verification study has been conducted in order to evaluate the accuracy of the simulation results and the main sources of numerical uncertainty. All the simulation results were used to achieve a surrogate model of lift and drag coefficients. This model is an effective mathematical tool for the preliminary design of Flettner rotor. Finally, an example of assessment of the Flettner rotor performance as an auxiliary propulsion device on a real tanker ship is reported.
\end{abstract}

\section{Introduction}

In the era in which most of the world's attention is focused on improving energy savings, the use of spinning cylinders as an auxiliary naval propulsion system has become a reality. Flettner rotors are rotating cylinders that, when immersed in a fluid stream, are able to produce fluid dynamic lift using the Magnus effect. This idea is due to the German engineer Anton Flettner who studied in the 1920s the effectiveness of spinning cylinders as a ship's propulsion system. This kind of propulsion systems was enrolled for the first time in 19251926 on the Buckau ship, shown in Figure 1(a). This ship used two rotors to augment the propulsion power of its former conventional sailing rigs. However, further commercial development of the FRs did not take place before the twentyfirst century. Nowadays, with increasing fuel prices and a general growing sensibility about green and environmentalprotection policies, the FRs are being seriously reconsidered as viable green ship propulsion devices. In 2010, Enercon, a wind energy company, launched a Flettner-powered cargo ship named E-Ship 1, Figure 1(b). On E-Ship 1 the FRs are used to assist the diesel engine: as reported in the Enercon technical report [1], the owner declares that thanks to this device a $30 \%$ reduction of fuel consumption is achieved. EShip 1 is in current use and has been recounted to cover more than 17000 sea miles with no mention of particular problems about the FRs [1].

\section{Literature Overview}

The capability of infinite length rotating cylinders to produce aerodynamic forces was studied for the first time at the Langley NACA Laboratory by Reid [2]; he found that in particular conditions such simple devices are capable of developing very high values of the lift coefficient and of the aerodynamic efficiency (i.e., the lift-to-drag ratio). Thom [3] presented an experimental work on rotating cylinders, with emphasis on the effects of the Reynolds number (Re), surface conditions, aspect ratio, and end plates disks, describing the device in terms of lift, drag, and torque coefficients. Successively, Swanson [4] clarified the physics underpinning the FR, thus highlighting the nature of the circulation around the rotating cylinder. More recently, a comprehensive study 


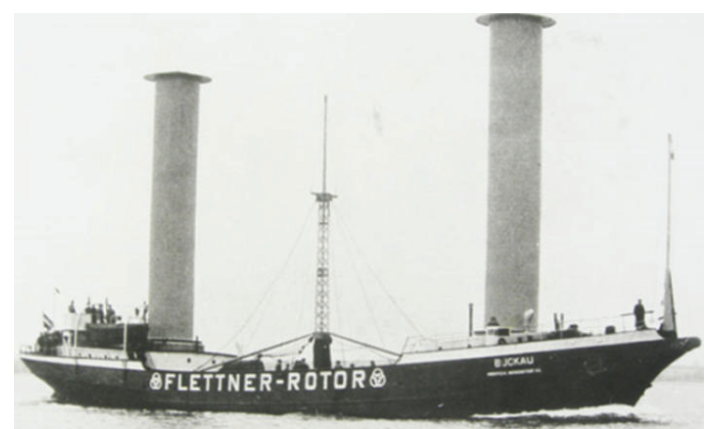

(a)

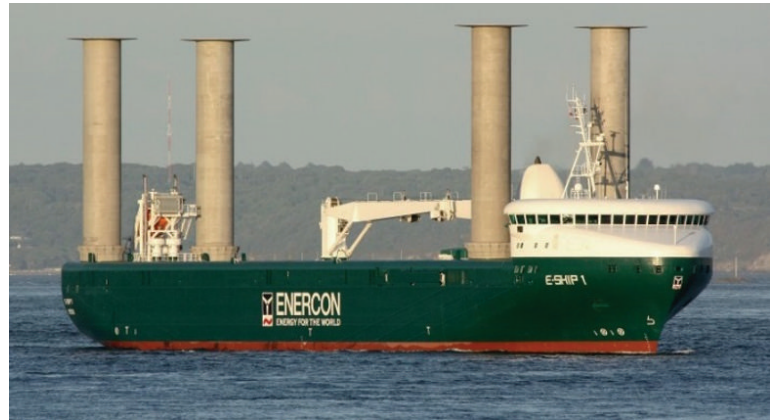

(b)

FIGURE 1: Buckau, first Flettner's ship (a), and E-Ship 1 by Enercon wind company (b).

on the functioning of FR was conducted by Da-Qing et al. [5]. This work presents a numerical study of aerodynamic performance of Flettner rotors at a high $\operatorname{Re}\left(1.6 \cdot 10^{6}\right)$ in relation to the change of spin ratio (SR) and aspect ratio (AR). Special attention is paid to the formation of vortex structures and the relationship between wake instability and fluctuation of aerodynamic loads. This paper also reports statistical expressions correlating $C_{L}$ and $C_{D}$ with SR.

The aerodynamic coefficients of an FR depend on various parameters (geometrical and functional). In the following subsections the most important parameters are briefly summarized.

2.1. Spin Ratio. The amount of aerodynamic force generated by a rotating cylinder, that is, an FR, is mainly dependent on the SR (or velocity ratio, also named $\alpha$ ), which accounts for the angular speed $\Omega$, the FR diameter $d$, and the free stream velocity $U$, as shown in Figure 2. The flow phenomena around a 3D circular cylinder are rather complex and feature both tip vortices and an alternate vortex shedding between the rotor sides. Seifert [6] highlights that vortex shedding occurs for Re all the way up to at least $8.0 \cdot 10^{6}$ and that the extension of the vortices depends also on the Strouhal number (St). The St represents the degree of unsteadiness of the oscillating flow past the rotating cylinder. In Mittal and Kumar [7], a detailed analysis of St regimes for rotating cylinders is presented. Low Strouhal numbers $\left(\mathrm{St}<10^{-4}\right)$ indicate long eddy formations; hence, the flow is considered quasi-steady. According to Badalamenti and Prince [8], the shedding phenomena are also influenced by SR: small spin ratios cause long eddy formations, while higher SRs cause considerably short eddies. Thus, the Kàrman vortex street is seen for SR $\leq 2$ when large eddies are formed and shed alternately on the two sides of the cylinder. Conversely, vortex formation and shedding can no longer be seen for $2.0 \leq \mathrm{SR}<$ 3.0 , and for $3.0 \leq \mathrm{SR}<3.5$ quasi-steady-states are observable. At $\mathrm{SR}=3.5$ a second shedding mode is found.

Swanson [4] pointed out that lift and drag of a rotating cylinder at $S R<1.0$ show a significant dependency on Re. The effects of Re are more evident on lift at Re $>6 \times 10^{4}$, which is also confirmed by Gowree and Prince [9]. When SR > 2.5

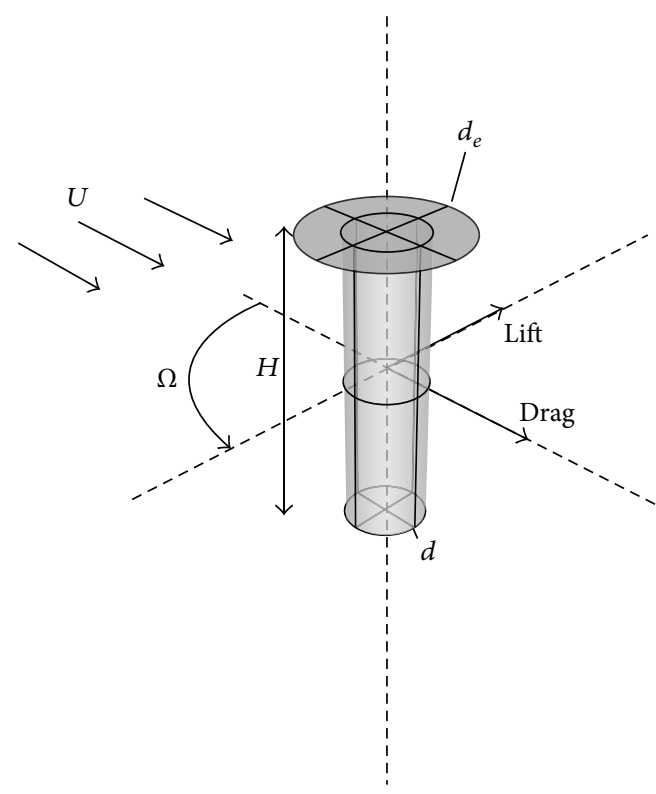

FIGURE 2: Sketch of the FR with end plate and main relevant parameters.

and $\operatorname{Re}>4 \times 10^{4}$, drag and lift curves have a slightly growing trend for increasing Re.

2.2. Aspect Ratio. The main shape factor of an FR is the aspect ratio $A R$, which significantly influences the FR effectiveness in producing aerodynamic forces. The AR of FR device, which is the ratio between height and diameter, modifies its aerodynamic efficiency, as for higher aspect ratios the FR device behaves much like a wing, with tip vortices that take part in the lift production. An important consideration is presented by Swanson [4], who observed that the smaller the aspect ratio the smaller the maximum lift obtained and the smaller the velocity ratio at which this maximum is reached. Swanson also demonstrated that, for very high aspect ratio, the lift can reach higher values than the maximum theoretical limit predicted by Prandtl's classical theory [10]. An extensive comparison of the main data available in the literature on FR 
performances, both experimental and numerical ones, along with some considerations, can be found in De Marco et al. [11].

2.3. End Plate. The idea of applying an end plate on FR to optimize its aerodynamic efficiency was first suggested by Prandtl [10]. The presence of an end plate modifies the $3 \mathrm{D}$ flow phenomena at the tip of the FR, augmenting the "effective AR" of the rotor. Thom [3] investigated the effect of large end plates with a diameter ratio $d_{e} / d=3.0$, where $d_{e}$ is the diameter of the end plate disk (Figure 2).

The FR with end plate, also called Thom disk, is able to produce almost double the lift at high velocity ratios; for example, $\mathrm{SR}=2.0$. In Badalamenti and Prince [12] it is shown that for a cylinder with $\mathrm{AR}=5.1$ and diameter ratios ranging from 1.1 to 3.0 , the effects of the increases $d_{e} / d$ and $\mathrm{AR}$ are similar. Increasing the $d_{e} / d$ value, a higher lift value is generated by the FR and such value occurs at higher SR as well (see Seifert [6]). Thouault et al. [13] presented a detailed analysis of the effects of end plate dimensions on the FR efficiency, based on experiments and numerical simulations.

A discussion of how the end plate size is related to SR in order to achieve optimal performances of the FR is presented by Seifert [6]. In his work Seifert observes that at low spin ratio $(\mathrm{SR}=1.0)$ smaller plates generally give lightly smaller drag; for applications at moderate spin ratio $(1.0<\mathrm{SR}<$ 3.0) larger plates are preferred, so as to delay the increase in induced drag, while, for high spin ratio applications (SR > 3.0), smaller plates are again more desirable.

2.4. Marine Application of FR. Concerning the use of FR for marine applications, not many research papers are available in the literature. An overview of the applications of the Magnus effect devices in the marine field is given in Morisseau [14]. The Magnus effect devices can be used as roll stabilizers, water propellers, and air generator, such as FR. Moreover this paper reports an interesting preliminary analysis of retrofitting of a single screw US Navy auxiliary ship with five FRs. More recently, in Pearson [15], a "firststage" assessment is found in practical limitations as well as negative side effects of retrofitting Flettner rotors to a ship. All these considerations are collected in order to create a software model for a preliminary analysis of the viability of retrofitting FR to a defined ship, before any progression onto analyzing specific scenario benefits or other detailed investigations. A limit of this work is in the estimation of FR performances, which are evaluated only as a function of SR. The software presented assumes a universal FR geometry, with $d_{e} / d=1.5$ and $\mathrm{AR}=5$ (based on Prandtl's study [10]) and evaluates $C_{L}$ and $C_{D}$ of this FR; then, the performances of FR are evaluated changing $S R$ in the range $0.0 \sim 8.0$.

Traut et al. [16] explore the potential for harnessing wind power for shipping applications. Numerical models of the two main wind power technologies, FR and towing kite, are linked with wind data along a set of five trade routes. The results of their analysis give an estimation of the average wind power contribution on a defined route. For a single FR, the delivered power is in a range between 193 and $373 \mathrm{~kW}$ and,

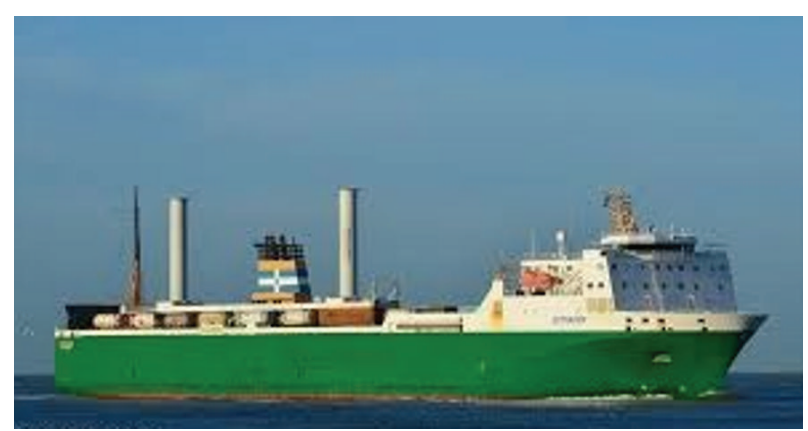

FIGURE 3: Estraden ship: latest FR installation.

for the towing kite, between 127 and $461 \mathrm{~kW}$. The FR has a variability of the delivered power smaller than the towing kite, due to the different dependencies on wind speed and direction. The average power contribution coming from an FR is higher than that coming from the kite on some routes and lower on others. But an advantage of FR is that the contribution would be expected to increase almost linearly with the number of devices installed on the same ship (in this respect, a quantitative study of interference effects is lacking in the literature). For this reason, for instance, installing three FRs on a 5500 DWT (dead weight tonnage) general cargo carrier could provide, on average, more than half the power required by the main engine under typical slow steaming conditions.

2.5. Aim of the Work. The present research extends previous investigations presented in De Marco et al. [17] and in De Marco et al. [11] in order to assess, in a systematic way, the effect of the FR key parameters, that is, the AR, SR, and the end plate diameter, on the device performance. Furthermore, the mutual interaction effects of these parameters on the aerodynamic forces generated by FR are investigated. The analyzed ranges of variation of the key parameters have been chosen considering technologically plausible marine applications of the FR concept.

\section{Flettner Rotor Installations and Reference Data}

To choose the ranges of the key parameters some constraints have been taken into account. For instance one has to consider the currently available technology as well as the practicality in marine applications. Another important factor is the vortex shedding risk (first and second mode) which depends on the mutual interaction between AR and SR. Consequently the limits of AR and SR have been identified by the analysis of some real FR installations on board.

The known installations of FR and their reference data are summarized in Table 1. The first two examples, Buckau and Barbara, are not in service, while the two ships, EShip-1 and Estraden (Figure 3), are currently operated by North European owners for commercial purposes. In all of these examples a Thom disk is used and the AR are in the range 5.5 7.0. This range is taken as a reference for the 
TABLE 1: Geometric, performance, and structural related parameters collected from all-known rotor ships.

\begin{tabular}{lcccc}
\hline Ship (year) & Buckau (1924) & Barbara (1926) & E-Ship 1 (2010) & Estraden (2014) \\
\hline Type & Retrofit & Newbuild & Newbuild & Retrofit \\
Height $(\mathrm{m})$ & 15.6 & 17.0 & 27.0 & 19.0 \\
Diameter $(\mathrm{m})$ & 2.8 & 4.0 & 6.8 & 3.0 \\
Aspect ratio & 5.6 & 4.3 & Yes & 6.3 \\
End plate & Yes & Yes & NA & Yes \\
Material & Zinc coated steel & Aluminum & NA & Composite \\
Max rpm & 135.0 & 150.0 & 250.0 \\
\hline
\end{tabular}

TABLE 2: Values of analyzed variables.

\begin{tabular}{lcccc}
\hline Variables & \multicolumn{5}{c}{ Values } \\
\hline SR & 1.0 & 1.5 & 2.0 & 3.0 \\
$\mathrm{AR}$ & 2.0 & 4.0 & 6.0 & 8.0 \\
$d_{e} / d$ & 1.0 & 2.0 & 3.0 & $/$ \\
\hline
\end{tabular}

investigations presented here. It is observed that the angular rotor speeds have increased over the years, thus allowing higher SR. However the SR values of interest for marine application remain in the range 1.0 to 3.0. For example, $\mathrm{SR}=$ 2.5 is obtained for a typical relative wind velocity of $20 \mathrm{kn}$ $(10 \mathrm{~m} / \mathrm{s})$, a reasonable diameter of $3.0 \mathrm{~m}$, and a rotation speed of $160 \mathrm{rpm}$.

\section{Numerical Experiments}

Systematic variations of FR configurations have been investigated by means of URANS simulations in incompressible flow. All the analyses have been performed using the commercially available computational fluid dynamics software CD adapco STAR-CCM+ v. 9.06. The simulations were conducted using the same approach, such as the overset/chimera grid technique, described in De Marco et al. [17] and in De Marco et al. [11]. Furthermore fairly wide ranges of the key parameters, that is, $\mathrm{AR}, \mathrm{SR}$, and $d_{e} / d$, have been examined, as detailed in the Table 2 and displayed in Figure 4. The characteristics of FRs were evaluated in terms of lift and drag coefficients and aerodynamic efficiency. The simulations have been launched on the computer cluster of the SCoPE supercomputing centre at the University of Naples "Federico II," using up to 120 CPUs in parallel.

4.1. Simulation Setup. The rotating motion of the FR was simulated using the overset/chimera mesh methodology with distance-weighted interpolation method. This method, which is especially suitable for rotational movements, uses an interpolation factor inversely proportional to the distance from acceptor cell to donor cell, as indicated in CD adapco User's Guide [18].

Hybrid mesh approach, coupling unstructured and structured mesh, has been used for all the simulations. The computational domain contains two regions: the background, nonrotating, region and the overlapped, rotating, region (Figure 5). For the background and overlapped region, an

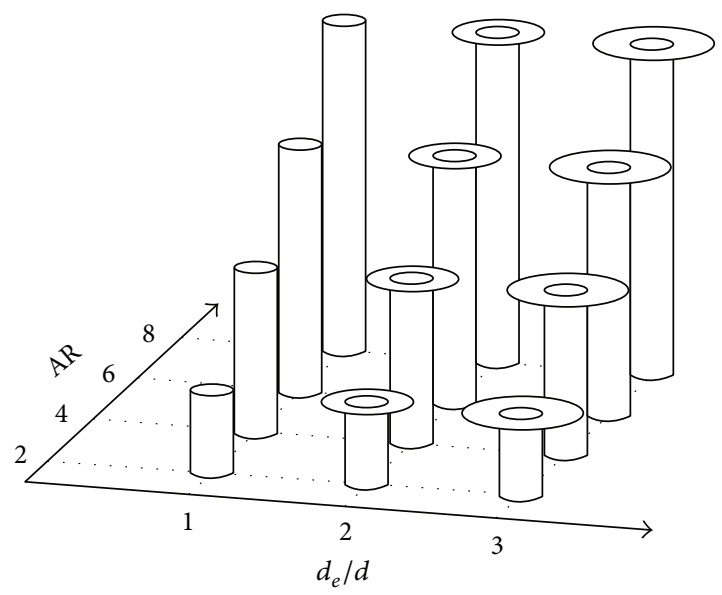

FIGURE 4: Sketch of all geometry of FRs tested.

unstructured grid approach was used, while a structured boundary layer mesh was created near the FR surface. The choice of hybrid mesh approach is justified by the fact that this is a suitable compromise between accuracy and computational effort compared with the Cartesian mesh, as shown in the 2D preliminary study reported in De Marco et al. [11]. Furthermore the grid set up allowed a nondimensional wall distance $(y+)$ value approximately equal to 1.0.

The chosen URANS-solving algorithm uses a first-order forward Euler scheme for the temporal discretization, an implicit element-based finite volume method, and a segregated flow approach with second-order upwind discretization of the convective terms. A fully turbulent approach with $k-\omega$ Shear Stress-Transport (SST) turbulence model has been used (a comparison with other turbulence model is reported in Figure 9). All of the properties of the numerical solver are summarized in Table 3.

It has to be noted that the simulation time step is a function of the angular speed $\Omega$. For the convergence of the numerical scheme, as a rule of thumb, there is a limitation on the maximum cell-based Courant-Friedrichs-Lewy (CFL) number in a time step. Therefore, the higher the angular speed the lower the time step.

4.2. Computational Domain and Boundary Conditions. A box-shaped domain has been created around the cylinder 
TABLE 3: Summary of the numerical simulation setup.

\begin{tabular}{|c|c|c|c|c|c|c|c|}
\hline Pressure link & Pressure & $\begin{array}{l}\text { Convection } \\
\text { term }\end{array}$ & $\begin{array}{c}\text { Temporal } \\
\text { discretization }\end{array}$ & Time step (s) & $\begin{array}{l}\text { Iteration per } \\
\text { time step }\end{array}$ & $\begin{array}{c}\text { Turbulence } \\
\text { model }\end{array}$ & $\begin{array}{c}\text { Overset } \\
\text { interpolation } \\
\text { scheme }\end{array}$ \\
\hline Simple & Standard & 2nd order & 1st order & $\begin{array}{c}\text { Function of } \\
\text { angular speed } \\
(\Omega)\end{array}$ & 11 & $k-\omega \mathrm{SST}$ & $\begin{array}{l}\text { Distance } \\
\text { weighted }\end{array}$ \\
\hline
\end{tabular}

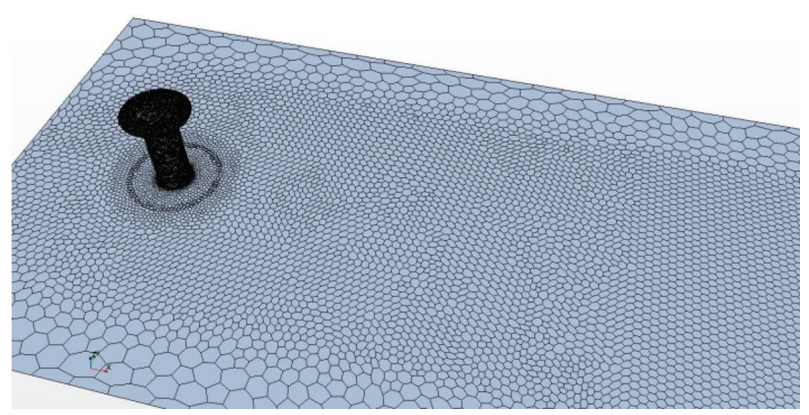

(a)

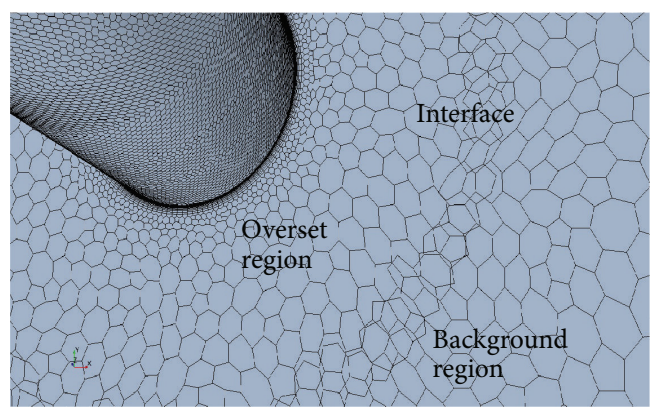

(b)

FIGURE 5: Section of the computational grid (a). Close-up views of the hybrid mesh (b).

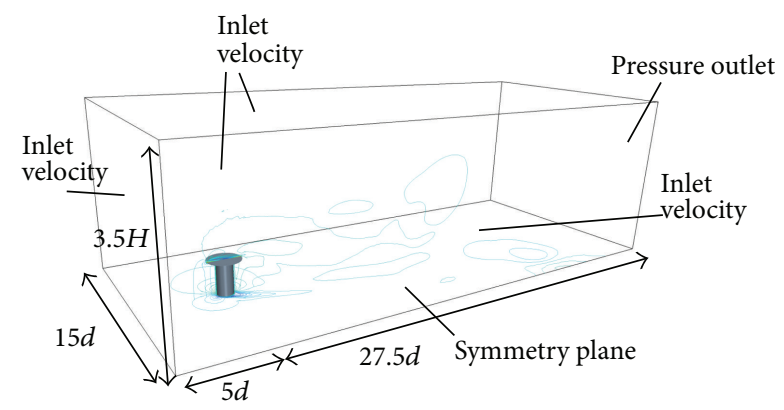

FIGURE 6: Boundary conditions and domain dimensions in function of the main dimensions of $\mathrm{FR}(H, d)$.

geometry, as seen in Figure 6. In order to reduce the computational effort, only half the domain has been considered and a symmetry plane has been assumed, containing the cylinder axis and parallel to the free stream velocity. A velocity inlet boundary condition has been set on the front side of the domain with a prescribed velocity: this inlet velocity also has been used to control the SR of the rotor, keeping constant its angular speed $\Omega$. On the bottom and the top side of the domain, a symmetry boundary condition also is used. On the rear side of the domain surface, a pressure outlet boundary condition has been imposed with a relative pressure of value $0 \mathrm{~Pa}$. An axial-symmetrical zone surrounding the rotor had to be modeled, overlapping the rotating mesh (fixed with the rotor) and the underlying nonmoving mesh domain. This turned out to be a necessary grid treatment for using the overset mesh methodology.

4.3. Numerical Uncertainty Analysis. In order to assess the numerical setup and to evaluate the simulation numerical uncertainty $U_{\mathrm{SN}}$, a verification study has been performed.
The benchmark experimental data are derived from Badalamenti and Prince $[8,12]$ and are related to an FR with $\mathrm{AR}=5.1$ (cylinder height $H=0.45 \mathrm{~m}$ ), diameter of $d=$ $0.0889 \mathrm{~m}$, and the Thom disk, on the top of this FR, with $d_{e}=0.1778 \mathrm{~m}$ (corresponding to $d_{e} / d=2.0$ ).

According to the Oberkampf and Blottner [19], verification is defined as a process for assessing simulation numerical uncertainty $U_{\mathrm{SN}}$. The simulation numerical error and uncertainty are composed of a grid convergence error $\left(\delta_{G}\right)$, iterative convergence error $\left(\delta_{I}\right)$, time step convergence error $\left(\delta_{\mathrm{TS}}\right)$, and other parameters $\left(\delta_{P}\right)$. Therefore the numerical error is the sum:

$$
\delta_{\mathrm{SN}}=\delta_{G}+\delta_{I}+\delta_{\mathrm{TS}}+\delta_{P}
$$

and the simulation numerical uncertainty is given by the formula

$$
U_{\mathrm{SN}}^{2}=U_{G}^{2}+U_{I}^{2}+U_{\mathrm{TS}}^{2}+U_{P}^{2}
$$

where $U_{G}, U_{I}, U_{\mathrm{TS}}$, and $U_{P}$ are the uncertainties arising from the grid, iterative, time step, and other parameters, respectively (see Stern et al. [20]).

The numerical uncertainty evaluation was performed using two different methods: the grid convergence index (GCI) method and the correction factor (CF) method. The general form of the uncertainty evaluation, based on the generalized Richardson extrapolation (RE) method, can be written as follows:

$$
U_{k}=F_{S}\left(\frac{\varepsilon_{21_{k}}}{r_{k}^{p_{k}}-1}\right),
$$

where $\varepsilon_{21_{k}}$ is the solution changes for the $k$-input parameter between the solutions (fine $\left(S_{1 k}\right)$ to medium $\left(S_{2 k}\right)$ and coarse $\left.\left(S_{3 k}\right)\right), r_{k}$ is the constant refinement ratio (recommended 
values between $\sqrt{ } 2$ and 2 ), $p_{k}$ is the observed order of accuracy, and $F_{S}$ is the safety factor. Furthermore, another parameter is the convergence ratio $\left(R_{k}\right)$, which provides information about the convergence/divergence of a solution. The $R_{k}$ value was determined by the following ratio:

$$
R_{k}=\frac{\varepsilon_{21_{k}}}{\varepsilon_{32_{k}}} .
$$

The two different solution verification methods used in this study differ in the choice of safety factor $\left(F_{S}\right)$.

The GCI method proposed by Roache $[21,22]$ is used extensively and it is recommended, for example, by the American Society of Mechanical Engineers (ASME) [23] and the American Institute of Aeronautics and Astronautics (AIAA) [24]. Roache recommended for careful grid studies (three or more grids analyzed) 1.25 as the $F_{S}$ value.

The other method used is the CF described in Stern et al. [20] that uses a variable value of $F_{S}$. In the CF method, unlike in the GCI method, the uncertainty of the error depends on how close the solutions are to the asymptotic range. The expressions to assess the uncertainties were reported by Wilson et al. [25].

The verification study has been carried out for the critical points of $\mathrm{SR}=2.0$ and $\mathrm{SR}=2.5$, in terms of $C_{D}$ estimation error.

The iterative uncertainties are estimated by the fluctuations of the time-history of the results in the last few periods, as indicated in Stern et al. [20]. Specifically, as reported in (5), $U_{I}$ are estimated by half the difference of the maximum value $\left(S_{U}\right)$ and the minimum value $\left(S_{L}\right)$ of the final time-history of the results:

$$
U_{I}=\left|\frac{1}{2}\left(S_{U}-S_{L}\right)\right|
$$

Then, the grid uncertainty has been evaluated by the following expressions, that is, (6) for the GCI method and (7) for the CF method:

$$
\begin{aligned}
& U_{k}=1.25 \cdot\left|\frac{\varepsilon_{21_{k}}}{r_{k}^{p_{k}}-1}\right| \\
& U_{k} \\
& = \begin{cases}{\left[9.6\left(1-C_{k}\right)^{2}+1.1\right]\left|\frac{\varepsilon_{21_{k}}}{r_{k}^{p_{k}}-1}\right|,} & \left|1-C_{k}\right|<0.125 \\
{\left[2\left|1-C_{k}\right|+1\right]\left|\frac{\varepsilon_{21_{k}}}{r_{k}^{p_{k}}-1}\right|,} & \left|1-C_{k}\right| \geq 0.125,\end{cases}
\end{aligned}
$$

where $F_{S}$ is equal to 1.25 and $C_{k}$ is the correction factor. Verification results using three systematically refined grids with the refinement ratio $\left(r_{G}\right)$ equal to $\sqrt{ } 2$ are shown in Table 5. The grid sizes range from $0.9 \mathrm{M}$ to $1.9 \mathrm{M}$ grid points and the three grids tested are shown in Table 4.

Because $0<R_{G}<1$, monotonic convergence is achieved for $C_{L}$ and $C_{D}$, where $R_{G}$ is convergence ratio for the grid, calculated according to (4). The uncertainty values are reported in Table 5.
TABLE 4: The three grids tested.

\begin{tabular}{lcc}
\hline & Grids & \\
\hline Grid A & Coarse & $0.933 \cdot 10^{6}$ \\
Grid B & Medium & $1.320 \cdot 10^{6}$ \\
Grid C & Fine & $1.867 \cdot 10^{6}$ \\
\hline
\end{tabular}

The values of simulation uncertainty reported in Table 5 show that $U_{\mathrm{SN}}$ for $C_{D}$ is higher than $U_{\mathrm{SN}}$ for $C_{L}$, due to much larger errors in the estimation of $C_{D}$ than of $C_{L}$ for $\mathrm{SR}=2.0$ and $\mathrm{SR}=2.5$.

Iterative convergence is achieved for all simulations and $U_{I}$ is found to be negligible with respect to grid errors, similarly to what happens in other engineering applications (e.g., Wilson et al. [26] and Xing et al. [27]).

Moreover, the verification procedure cannot be completed with the validation phase due to the lack of experimental uncertainty data. Therefore, only the results related to simulation numerical uncertainty are reported in Table 5.

The numerical results of the verification study have been compared with the experimental data, as shown in Figure 7. The comparison highlighted that no significant improvement in the $C_{L}$ and $C_{D}$ evaluation is detected between coarse, medium, and fine mesh case. However, increasing the grid points increases the calculation effort, as shown in Figure 8.

For the reasons mentioned above, the grid of Case B has been assumed as the reference mesh. This grid guarantees an acceptable solution without an excessive computational effort (such as in Case C).

The comparison between experimental data and numerical results for $\mathrm{SR}$ in the range from 0.0 to 2.5, reported in Figure 9, shows a good agreement in particular for $C_{L}$. Regarding $C_{D}$, the CFD simulations give reliable results for $\mathrm{SR}<1.5$ and the error increases at higher values of SR, likewise highlighted in Thouault et al. [13] and Da-Qing et al. [5].

As shown in Table 5, it can be observed that for $C_{D}$ the comparison error $(E)$ is much greater than $U_{\mathrm{SN}}$. According to Oberkampf and Blottner [19] and Stern et al. [20], it can be said that when $E$ is much greater than the uncertainty, it is necessary to improve the simulations models. It is well known that one of the main sources of the modeling simulation error is the turbulence models. Nevertheless, as highlighted in Figure 9, comparing the different two-equation turbulence models, that is, $k-\omega$ SST and Realizable $k-\varepsilon$, no significant differences are appreciable. The $k-\omega$ SST is used as turbulence model for all the subsequent simulations; however a different simulation approach is required for an accurate $C_{D}$ evaluation at high SR.

\section{Results}

The results of the simulations, performed into the variable ranges indicated in Table 2, are reported in terms of the socalled response curves. These curves, shown in Figure 10, represent the values of $C_{L}$ and $C_{D}$ and aerodynamic efficiency, keeping constant $d_{e} / d$ of the FR. This representation is useful because it permits us to clearly recognize the relationships 


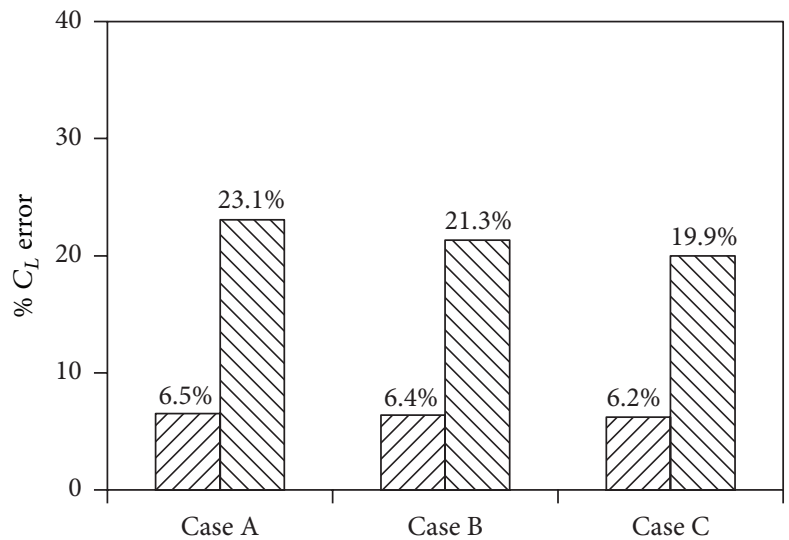

$\oslash$ SR 2.5

$\triangle$ SR 2.0

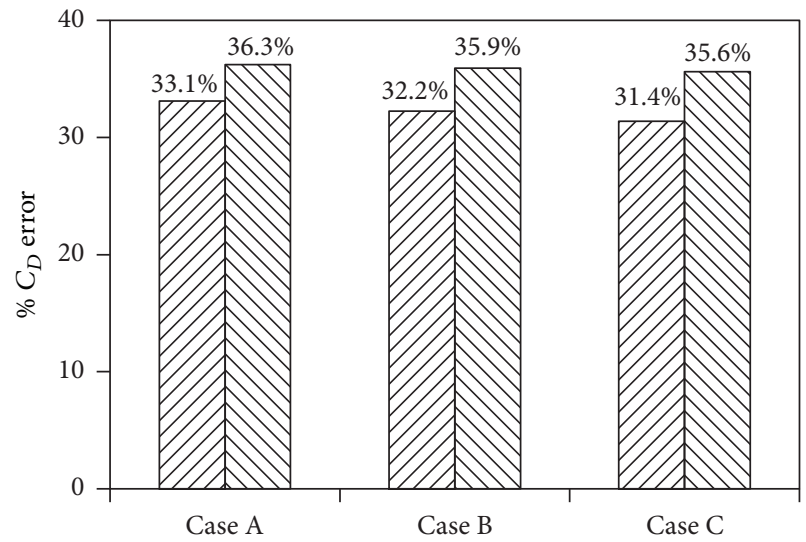

$\nabla$ SR 2.5

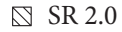

(a)

(b)

Figure 7: $C_{L}$ and $C_{D}$ percentage error between numerical (three mesh cases tested) and experimental data at $\mathrm{SR}=2.0$ and 2.5 .

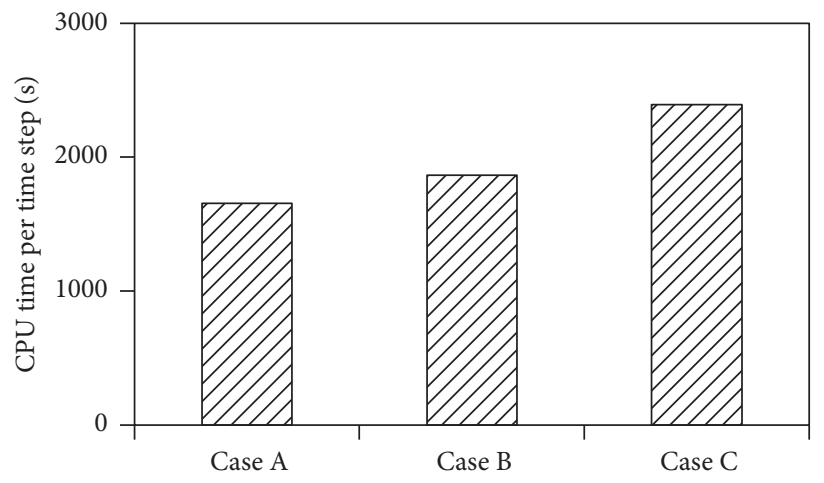

FIGURE 8: Computational time required for the grids tested.

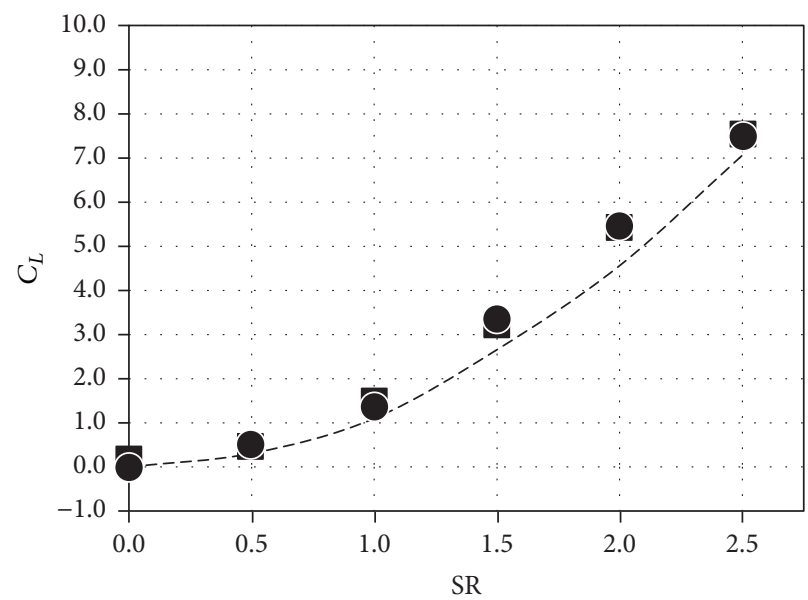

--- Exp data

CFD $k-\varepsilon$

- CFD $k-\omega$ SST

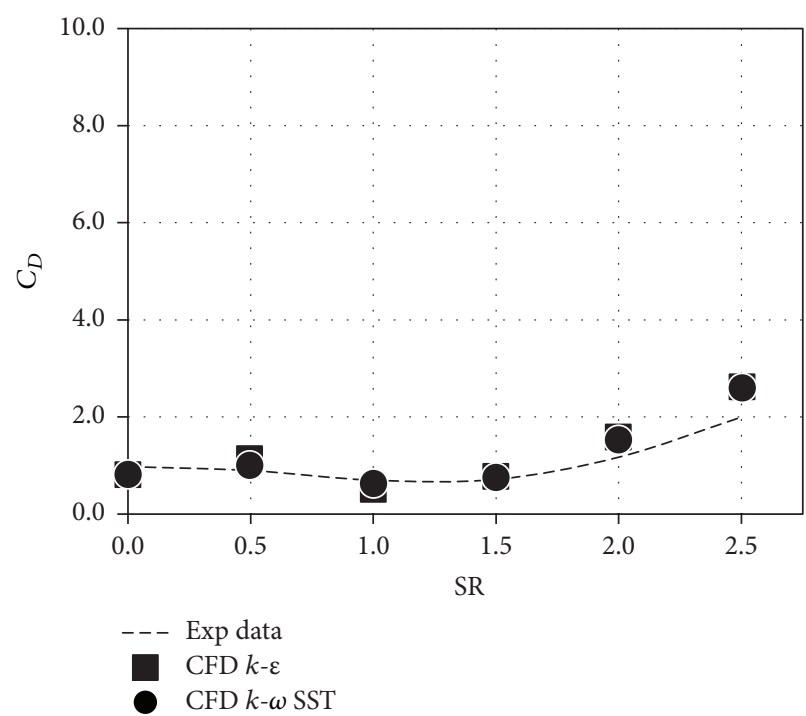

(b)

Figure 9: Comparison between CFD results and experimental data for lift (a) and drag (b) coefficient using mesh Case B and two different turbulence models. 
TABLE 5: Grid and iterative uncertainty for $C_{L}$ and $C_{D}$ at the two different SRs.

\begin{tabular}{|c|c|c|c|c|c|c|c|c|c|c|c|}
\hline & SR & Grids & Grid ratio & $R_{G}$ & $P_{G}$ & $1-C_{G}$ & $\begin{array}{c}\% U_{G} \\
\mathrm{GCI}\end{array}$ & $\begin{array}{c}\% U_{G} \\
\mathrm{CF}\end{array}$ & $\% U_{I}$ & $\% U_{\mathrm{SN}}$ & $\%|E|$ \\
\hline \multirow{2}{*}{$C_{L}$} & 2.0 & A-B-C & $\sqrt{ } 2$ & 0.80 & -0.65 & 1.20 & 8.91 & 4.26 & 0.26 & 8.91 & 19.30 \\
\hline & 2.5 & $A-B-C$ & $\sqrt{ } 2$ & 0.96 & -0.12 & 1.04 & 4.40 & 3.23 & 0.55 & 4.43 & 6.24 \\
\hline \multirow{2}{*}{$C_{D}$} & 2.0 & $A-B-C$ & $\sqrt{ } 2$ & 0.97 & -0.04 & 1.01 & 20.06 & 15.60 & 1.37 & 20.11 & 36.23 \\
\hline & 2.5 & $A-B-C$ & $\sqrt{ } 2$ & 0.96 & -0.13 & 1.04 & 19.18 & 14.02 & 2.15 & 19.30 & 31.36 \\
\hline
\end{tabular}

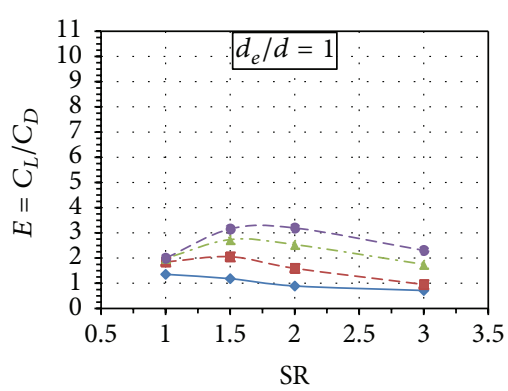

$\rightarrow \mathrm{AR}=2 \quad \rightarrow-\mathrm{AR}=6$

$\mathrm{AR}=4$

(a)

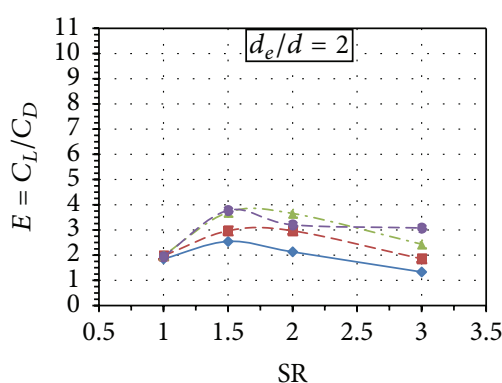

$\begin{array}{ll}-\mathrm{AR}=2 & -\mathrm{AR}=6 \\ -\mathrm{AR}=4 & -\mathrm{AR}=8\end{array}$

(d)

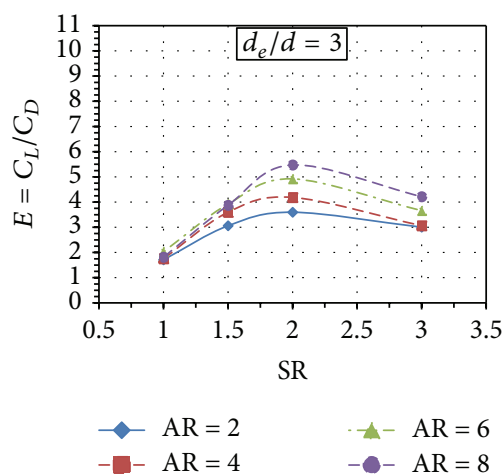

(g)

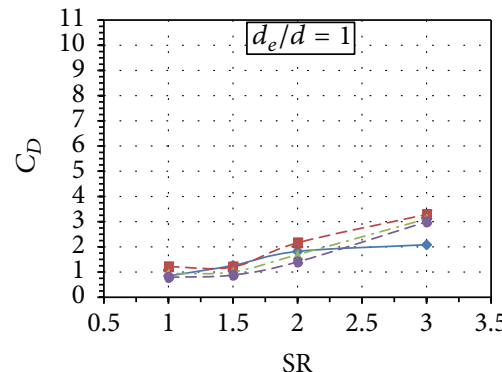

SR

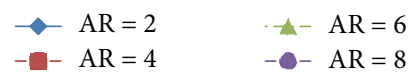

(b)

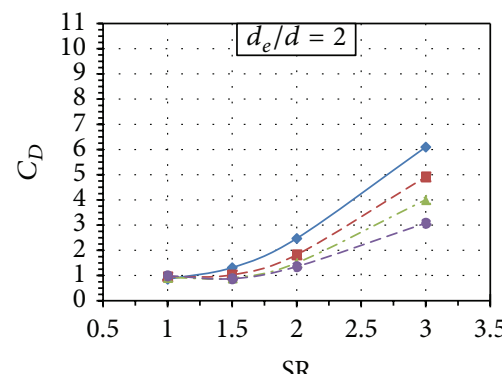

$-\mathrm{AR}=6$

- $\mathrm{AR}=8$

(e)

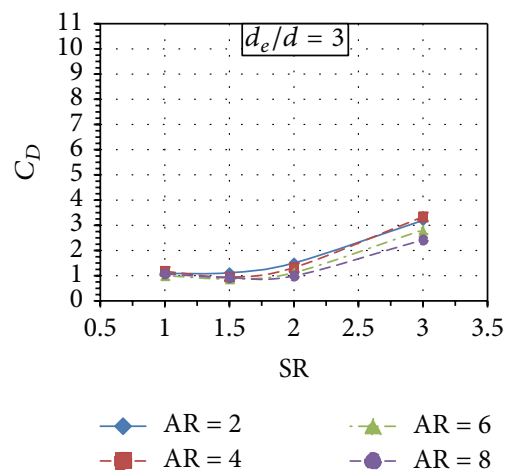

(h)
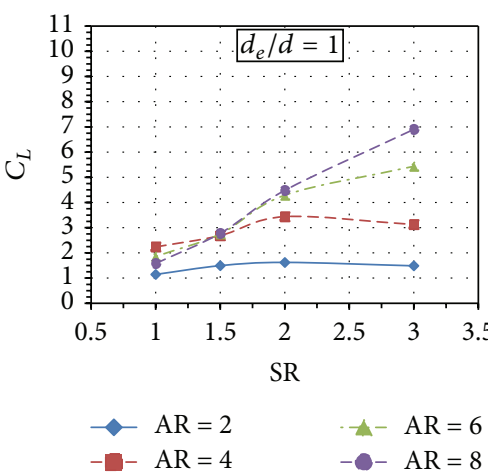

(c)

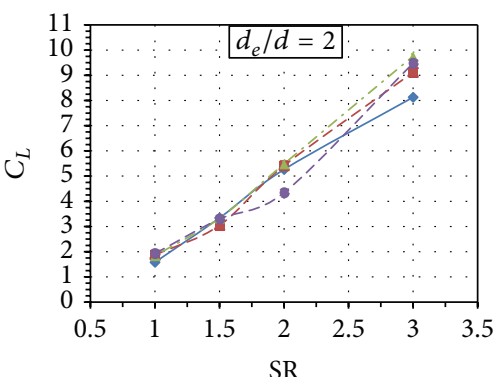

$\triangle-A R=6$

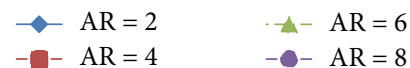

(f)

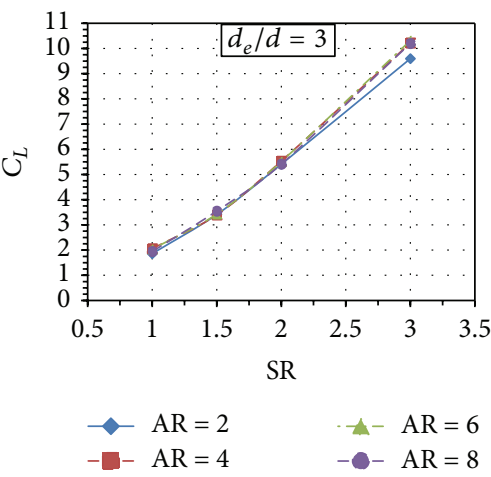

(i)

Figure 10: Response curves for $C_{L}, C_{D}$ and aerodynamic efficiency $(E)$ at various SR, AR, and $d_{e} / d$ for FR.

between geometrical (AR, $d_{e} / d$ ) and functional parameters (SR) with FR performances $\left(C_{L}, C_{D}\right.$, and aerodynamic efficiency).

The response curves in Figure 10 show that $C_{L}$ is mainly influenced by the SR of the FR: as expected, high values of
SR cause large value of $C_{L}$ due to higher circulation around the cylinder. Keeping constant the value of $d_{e} / d$, the amount of $C_{L}$ increases for high value of $\mathrm{AR}$, but this trend reduces for high values of $d_{e} / d$. Also, simulations confirm beneficial effects of the FR's end plate on its performance. Referring to 
$C_{D}$ the ability of larger end plates to reduce the induced drag component is confirmed, as shown in Figure 10. The reason for these improvements can be due to an effect similar to increasing the $\mathrm{AR}$ values.

About the variation of $C_{D}$ with $\mathrm{SR}$, this has the same behavior of $C_{L}$, while $C_{D}$ decreases as AR increases, as for a typical aircraft wing. The maximum absolute value of the FR aerodynamic efficiency rises and translates to higher SR values, when $\mathrm{AR}$ and $d_{e} / d$ increase too.

\section{Surrogate Model for the Lift and Drag Coefficients}

$C_{L}$ and $C_{D}$ equations in (8) summarize the results of the extensive numerical analyses on the behavior of FR. These equations represent a surrogate model which can be used to predict the performance of the FR in relation to SR, AR, and $d_{e} / d$. This model is an effective mathematical tool for the preliminary design of FR.

The ratio of these two formulas allows evaluating the aerodynamic efficiency of such devices. In both equations, the coefficients $a_{i j k}$ and $b_{i j k}$ transform geometrical and functional FR's parameters into $C_{L}$ and $C_{D}$. The values of $a_{i j k}$ and $b_{i j k}$ coefficients are presented in Table 6 . The coefficients of the matrices $a_{i j k}$ and $b_{i j k}$ have been obtained by applying a leastsquares root fit procedure to the numerical results, similar to the optimization techniques used to find a set of design parameters, as described in Balsamo et al. [28]:

$$
\begin{aligned}
C_{L} & =\sum_{i=1}^{4} \sum_{j=1}^{4} \sum_{k=1}^{3} a_{i j k} \mathrm{SR}^{i} \mathrm{AR}^{j}\left(\frac{d_{e}}{d}\right)^{k} \\
C_{D} & =\sum_{i=1}^{4} \sum_{j=1}^{4} \sum_{k=1}^{3} b_{i j k} \operatorname{SR}^{i} \mathrm{AR}^{j}\left(\frac{d_{e}}{d}\right)^{k} .
\end{aligned}
$$

The validity of these equations is strictly related to the range of the variables analyzed: $1.0 \leq \mathrm{SR} \leq 3.0,2.0 \leq \mathrm{AR} \leq 8.0$, and $1.0 \leq d_{e} / d \leq 3.0$.

A test of the reliability of the surrogate model has been performed using the experimental data available in Pearson [15] relevant to FR with $\mathrm{AR}=5.0$ and $d_{e} / d=1.5$. These values were used as input for (8). The comparison between experimental data and the predicted results is shown in Figure 11 and it can be noted that $C_{L}$ is correctly estimated, while $C_{D}$ is overestimated for values of SR, which are greater than 2.5. This discrepancy, which is due to the higher uncertainty on the simulated drag results, is less critical in the perspective of marine applications. In fact as indicated above, the SR values of interest in this field are not larger than 3.0, often around 2.0 .

\section{Flettner Rotor as Ship Propulsion Device}

To evaluate the potentiality of the FRs as marine propulsion devices, it is important to consider that for FR on a ship the resulting wind speed is the vector sum of the environmental wind and the ship speed. If the resulting wind relative to the ship coordinate system is coming from the bow quarter

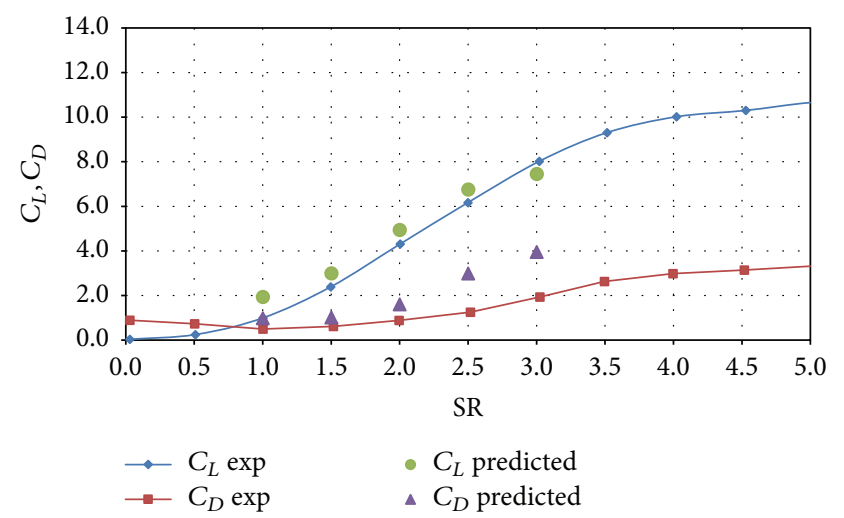

FIGURE 11: Comparison of experimental and predicted values of $C_{L}$ and $C_{D}$ for FR with $\mathrm{AR}=5.0$ and $d_{e} / d=1.5$.

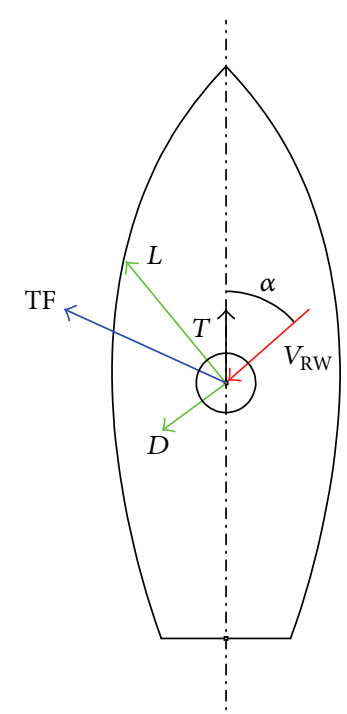

(a)

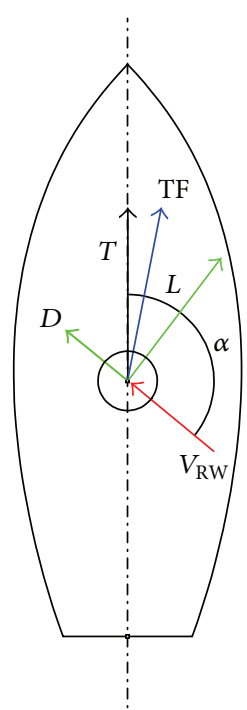

(b)
FIGURE 12: Total force and thrust delivered by FR at different apparent wind directions: from bow quarter (a), from stern quarter (b).

direction as illustrated in Figure 12(a), $D$ will have a negative contribution to the resultant thrust $(T)$. In this case, a lower drag will of course be beneficial. However, if the resulting wind is from the stern quarter direction as depicted in Figure 12(b), then $D$ also contributes to $T$ : under this circumstance a high drag is not disadvantage.

In Figures 12(a) and 12(b), TF is the resultant of $L$ and $D$, $V_{\mathrm{RW}}$ the relative wind velocity, $T$ the effective thrust, and $\alpha$ the apparent wind angle. Ultimately, for a geometrically welldefined FR, the resultant thrust depends on the relative wind velocity, on the apparent wind angle, and on the angular speed $\Omega$ of the FR. Obviously, the relation between $V_{\mathrm{RW}}$ and $\Omega$ is quantified by SR.

Figure 13 shows the values of $C_{T}$ and $T$ resulting from the polynomials whose coefficients are shown in Table 6.

As strongly suggested by Figure 13, the thrust has been evaluated at the maximum SR simulated in the study 
TABLE 6: The values of $a_{i j k}$ and $b_{i j k}$ coefficients reported in (8).

\begin{tabular}{|c|c|c|c|c|c|c|c|c|c|}
\hline$i$ & $j$ & $k$ & $a_{i j k}$ & $b_{i j k}$ & $i$ & $j$ & $k$ & $a_{i j k}$ & $b_{i j k}$ \\
\hline 0 & 0 & 0 & 46.756 & -79.919 & 1 & 0 & 0 & -89.785 & 140.718 \\
\hline 0 & 0 & 1 & -78.301 & 70.071 & 1 & 0 & 1 & 140.010 & -125.048 \\
\hline 0 & 0 & 2 & 20.282 & -15.268 & 1 & 0 & 2 & -35.672 & 27.377 \\
\hline 0 & 1 & 0 & -37.846 & 60.030 & 1 & 1 & 0 & 77.607 & -104.703 \\
\hline 0 & 1 & 1 & 60.058 & -50.611 & 1 & 1 & 1 & -112.204 & 89.137 \\
\hline 0 & 1 & 2 & -15.301 & 11.013 & 1 & 1 & 2 & 27.977 & -19.292 \\
\hline 0 & 2 & 0 & 8.096 & -11.649 & 1 & 2 & 0 & -16.461 & 20.215 \\
\hline 0 & 2 & 1 & -11.874 & 9.810 & 1 & 2 & 1 & 22.038 & -17.098 \\
\hline 0 & 2 & 2 & 2.987 & -2.141 & 1 & 2 & 2 & -5.409 & 3.698 \\
\hline 0 & 3 & 0 & -0.460 & 0.669 & 1 & 3 & 0 & 0.915 & -1.155 \\
\hline 0 & 3 & 1 & 0.639 & -0.558 & 1 & 3 & 1 & -1.152 & 0.964 \\
\hline 0 & 3 & 2 & -0.160 & 0.121 & 1 & 3 & 2 & 0.280 & -0.207 \\
\hline$i$ & $j$ & $k$ & $a_{i j k}$ & $b_{i j k}$ & $i$ & $j$ & $k$ & $a_{i j k}$ & $b_{i j k}$ \\
\hline 2 & 0 & 0 & 38.547 & -76.506 & 2 & 0 & 0 & -4.895 & 11.799 \\
\hline 2 & 0 & 1 & -62.545 & 69.271 & 2 & 0 & 1 & 8.642 & -10.539 \\
\hline 2 & 0 & 2 & 16.266 & -15.189 & 2 & 0 & 2 & -2.294 & 2.290 \\
\hline 2 & 1 & 0 & -37.556 & 57.951 & 2 & 1 & 0 & 5.190 & -9.412 \\
\hline 2 & 1 & 1 & 54.801 & -49.793 & 2 & 1 & 1 & -7.946 & 8.065 \\
\hline 2 & 1 & 2 & -13.704 & 10.709 & 2 & 1 & 2 & 2.016 & -1.716 \\
\hline 2 & 2 & 0 & 8.153 & -11.180 & 2 & 2 & 0 & -1.154 & 1.831 \\
\hline 2 & 2 & 1 & -10.753 & 9.493 & 2 & 2 & 1 & 1.556 & -1.547 \\
\hline 2 & 2 & 2 & 2.627 & -2.034 & 2 & 2 & 2 & -0.383 & 0.327 \\
\hline 2 & 3 & 0 & -0.440 & 0.636 & 2 & 3 & 0 & 0.060 & -0.104 \\
\hline 2 & 3 & 1 & 0.534 & -0.531 & 2 & 3 & 1 & -0.073 & 0.086 \\
\hline 2 & 3 & 2 & -0.128 & 0.113 & 2 & 3 & 2 & 0.018 & -0.018 \\
\hline
\end{tabular}

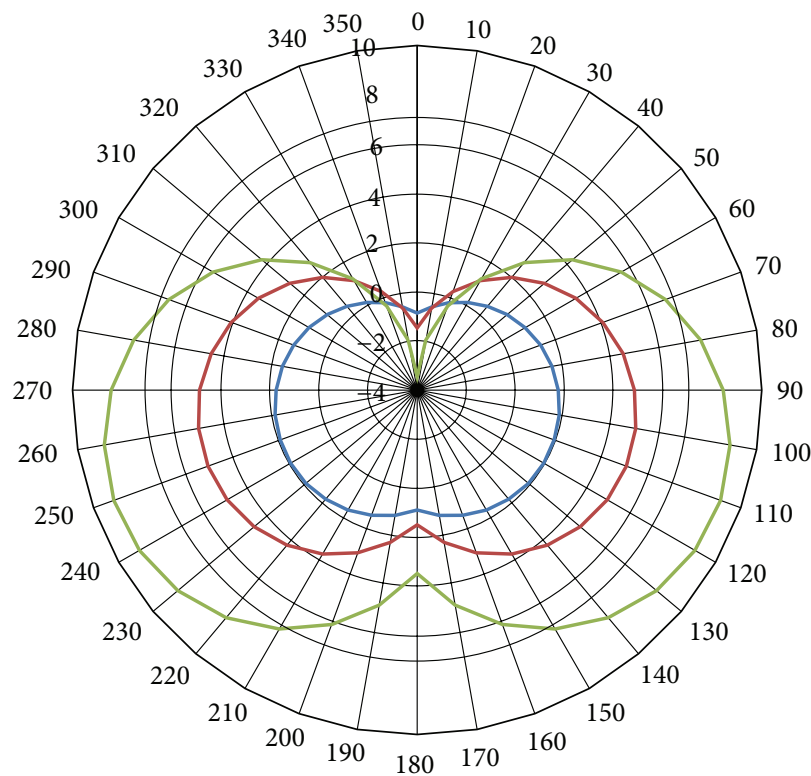

Thrust coefficient

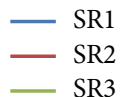

(a)

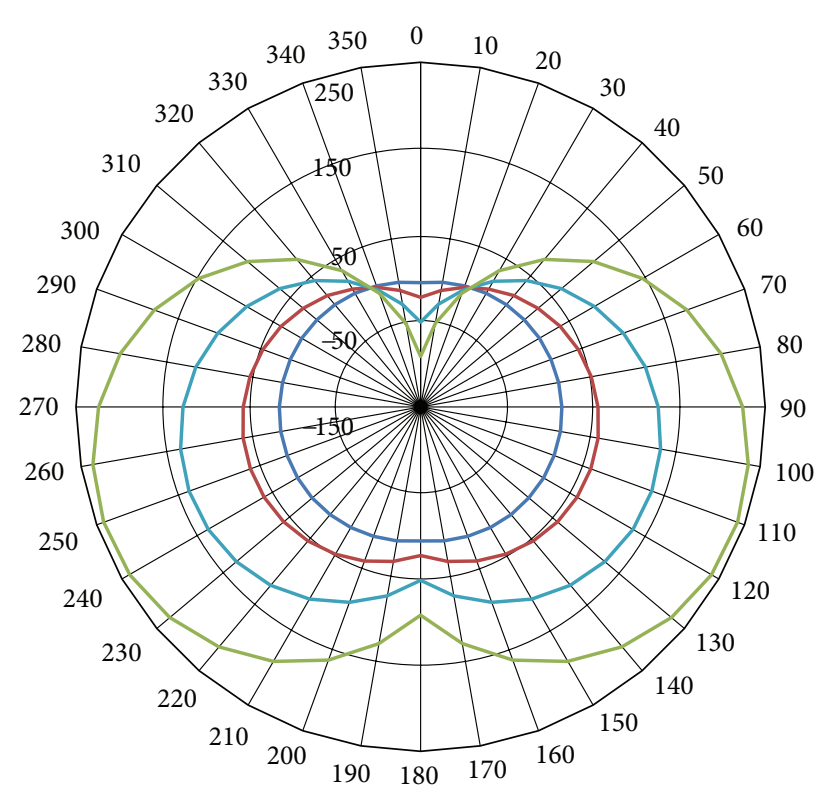

Thrust $(\mathrm{kN})$

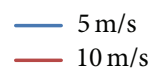

$-15 \mathrm{~m} / \mathrm{s}$

(b)

Figure 13: In (a) $C_{T}$ for a defined FR geometry $(H=28.0 \mathrm{~m}, d=4.0 \mathrm{~m})$ at different values of SR and apparent wind angle. In (b) thrust values delivered by the FR for different magnitude of relative wind velocity (fixed SR $=3$ ). 
$(\mathrm{SR}=3.0)$ and referring to FR whose diameter and height were $4.0 \mathrm{~m}$ and $28.0 \mathrm{~m}$, respectively. These dimensions are consistent with the ship taken into consideration: a product tanker $205 \mathrm{~m}$ long at waterline and dislocating $74983 \mathrm{t}$. Towing tank tests of this ship indicate that the resistance at $10 \mathrm{kn}$ and $12 \mathrm{kn}$ is $354 \mathrm{kN}$ and $500 \mathrm{kN}$, respectively.

Therefore the comparison of resistance of a ship with the thrust data, as shown in Figure 13, highlights that a couple of FRs, as few as $20 \mathrm{kn}$ of $V_{\mathrm{RW}}$, are able to give, in a wide range of angles of apparent wind, a thrust whose magnitude is 0.3 and 0.2 times the resistance of a ship at $10 \mathrm{kn}$ and $12 \mathrm{kn}$, respectively.

The aim of these considerations is a rough evaluation of the potentiality of the FR as a marine propulsion device. For a more comprehensive analysis of the FR effectiveness, more aspects have to be taken into account. These include, for instance, the asymmetric hydrodynamic flow condition produced by the transversal component of TF (heeling and drift angle), extra rudder drag due to the aerodynamic yaw moment, and the reduced efficiency of FR due to the ship motions.

\section{Conclusions}

In this paper, a systematic approach to identify the most influencing parameters on FR performance and the applicability of such device for marine application has been presented. Results from the numerical simulations have been used to achieve a surrogate model useful for preliminary FR designs.

The uncertainty analysis shows that the highest numerical uncertainty and error are for $C_{D}\left(U_{\mathrm{SN}}=20.1 \%, E=36.2 \%\right)$, with respect to $C_{L}\left(U_{\mathrm{SN}}=8.9 \%, E=19.3 \%\right)$. Furthermore, it has been observed that the main source of numerical uncertainty is due to the grid. The $C_{D}$ error and numerical uncertainty trend seems to be related to the limits of the turbulence models used, that is, $k-\omega$ SST and Realizable $k-\varepsilon$. Reasonably, the Large Eddy Simulation (LES) analysis should be used for an accurate evaluation of $C_{D}$, in particular at high SR.

Regarding the capability of FR to act as a propulsion device, it is confirmed that, in terms of magnitude, $C_{L}$ and aerodynamic efficiency of $F R$ is much higher than the values given by a wing of comparable aspect ratio.

Finally, the potentiality of the FR as a marine propulsion device, here evaluated on a tanker ship, highlights that, in a wide range of wind angles, a couple of FR can give a thrust whose magnitude is up to $30 \%$ of the ship resistance in the range of operational speed. Also, the assessment of the aerodynamic efficiency is less significant than the evaluation of each of the aerodynamic force components, as the drag gives a positive contribution to the thrust in a wide range of apparent wind angles.

\section{Symbology and Abbreviations}

$\begin{array}{ll}\alpha: & \text { Apparent wind angle }(\mathrm{deg}) \\ A: & \text { Reference area }(H * d)\left(\mathrm{m}^{2}\right)\end{array}$

\begin{tabular}{|c|c|}
\hline $\mathrm{AR}=H / d:$ & Aspect ratio \\
\hline$C_{D}=D / 0.5 \rho A U^{2}:$ & Drag coefficient \\
\hline$C_{L}=L / 0.5 \rho A U^{2}:$ & Lift coefficient \\
\hline$C_{T}=T / 0.5 \rho A U^{2}:$ & Thrust coefficient \\
\hline CFL number: & $\begin{array}{l}\text { Courant-Friedrichs-Lewy } \\
\text { number }\end{array}$ \\
\hline$C_{k}:$ & Correction factor \\
\hline CF method: & Correction factor method \\
\hline$D:$ & $\operatorname{Drag}(\mathrm{N})$ \\
\hline$d:$ & Cylinder diameter $(\mathrm{m})$ \\
\hline$d_{e}:$ & End plate diameter $(\mathrm{m})$ \\
\hline$\delta_{I}:$ & Iteration convergence error \\
\hline$\delta_{G}:$ & Grid convergence error \\
\hline$\delta_{\mathrm{TS}}:$ & Time step convergence error \\
\hline$\delta_{\mathrm{RE}}:$ & $\begin{array}{l}\text { Error evaluated by RE } \\
\text { method }\end{array}$ \\
\hline DWT: & Dead weight tonnage $(t)$ \\
\hline$\varepsilon_{k i j}:$ & Solution change \\
\hline$E:$ & Comparison error \\
\hline$f:$ & Frequency $(\mathrm{Hz})$ \\
\hline$F_{S}:$ & Factor of safety \\
\hline FR: & Flettner rotor \\
\hline GCI: & Grid convergence index \\
\hline$H:$ & Cylinder length (m) \\
\hline$L:$ & Lift $(\mathrm{N})$ \\
\hline LES: & Large Eddy Simulation \\
\hline$v:$ & Dynamic viscosity ( $\mathrm{Pa} \mathrm{s}$ ) \\
\hline$\Omega:$ & Angular velocity ( $\mathrm{rad} / \mathrm{s})$ \\
\hline$\rho:$ & Air density $\left(\mathrm{kg} / \mathrm{m}^{3}\right)$ \\
\hline$p_{k}:$ & Estimated order of accuracy \\
\hline $\operatorname{Re}=U d / v$ : & Reynolds number \\
\hline RE: & $\begin{array}{l}\text { Richardson extrapolation } \\
\text { method }\end{array}$ \\
\hline$r_{k}:$ & Refinement ratio \\
\hline $\mathrm{SR}=\Omega d / 2 U:$ & Spin ratio \\
\hline $\mathrm{St}=f d / U:$ & Strouhal number \\
\hline$T:$ & Effective thrust $(\mathrm{N})$ \\
\hline TF: & Total force $(\mathrm{N})$ \\
\hline$U:$ & $\begin{array}{l}\text { Free stream or flux velocity } \\
(\mathrm{m} / \mathrm{s})\end{array}$ \\
\hline$U_{G}:$ & Grid uncertainty \\
\hline$U_{I}:$ & Iteration uncertainty \\
\hline$U_{\mathrm{SN}}:$ & Simulation uncertainty \\
\hline$U_{\mathrm{TS}}:$ & Time step uncertainty \\
\hline URANS: & $\begin{array}{l}\text { Unsteady Reynolds averaged } \\
\text { Navier-Stokes }\end{array}$ \\
\hline$y+:$ & $\begin{array}{l}\text { Nondimensional wall } \\
\text { distance. }\end{array}$ \\
\hline
\end{tabular}

\section{Competing Interests}

The authors declare that they have no competing interests.

\section{Acknowledgments}

The authors gratefully acknowledge the availability of the Calculation Centre SCoPE of the University of Naples "Federico 
II" and thanks are due to SCoPE academic staff for the given support.

\section{References}

[1] Enercon Wind Company, "Enercon E-ship 1: a wind-hybrid commercial cargo ship," in Proceedings of the 4th Conference on Ship Efficiency, Hamburg, Germany, September 2013.

[2] E. G. Reid, "Tests of rotating cylinders," Techinical Notes NACA 209, 1924.

[3] A. Thom, "Effects of discs on the air forces on a rotating cylinder," Reports \& Memoranda 1623, Aerospace Research Council, 1934.

[4] M. W. Swanson, "The Magnus effect: a summary of investigations to date," Journal of Basic Engineering, vol. 83, no. 3, pp. 461-470, 1961.

[5] L. Da-Qing, M. Leer-Andersen, and B. Allenström, "Performance and vortex formation of Flettner rotors at high Reynolds numbers," in Proceedings of 29th Symposium on Naval Hydrodynamics, Gothenburg, Sweden, August 2012.

[6] J. Seifert, "A review of the Magnus effect in aeronautics," Progress in Aerospace Sciences, vol. 55, pp. 17-45, 2012.

[7] S. Mittal and B. Kumar, "Flow past a rotating cylinder," Journal of Fluid Mechanics, vol. 476, pp. 303-334, 2003.

[8] C. Badalamenti and S. A. Prince, "Vortex shedding form a rotating circular cylinder at moderate subcritical reynolds numbers and high velocity ratio," in Proceedings of the 26th Congress of International Council of the Aeronautical Sciences (ICAS '08), Anchorage, Alaska, USA, September 2008.

[9] E. R. Gowree and S. A. Prince, "A computational study of the aerodynamics of a spinning cylinder in a crossflow of high Reynolds number," in Proceedings of the 28th Congress of the International Council of the Aeronautical Sciences (ICAS '12), pp. 1138-1147, Brisbane, Australia, September 2012.

[10] L. Prandtl, "The Magnus effect and wind-powered ships," Naturwissenschaften, vol. 13, pp. 1787-1806, 1925.

[11] A. De Marco, S. Mancini, and C. Pensa, "Preliminary analysis for marine application of Flettner rotors," in Proceedings of the 2nd International Symposium on Naval Architecture and Maritime (INT-NAM '14), Istanbul, Turkey, October 2014.

[12] C. Badalamenti and S. A. Prince, "Effects of endplates on a rotating cylinder in crossflow," in Proceedings of the 26th AIAA Applied Aerodynamics Conference, Honolulu, Hawaii, USA, August 2008.

[13] N. Thouault, C. Breitsamter, N. A. Adams, J. Seifert, C. Badalamenti, and S. A. Prince, "Numerical analysis of a rotating cylinder with spanwise disks," AIAA Journal, vol. 50, no. 2, pp. 271-283, 2012.

[14] K. C. Morisseau, "Marine application of magnus effect devices," Naval Engineers Journal, vol. 97, no. 1, pp. 51-57, 1985.

[15] D. R. Pearson, "The use of flettner rotors in efficient ship design," in Proceedings of the Influence of EEDI on Ship Design Conference, London, UK, September 2014.

[16] M. Traut, P. Gilbert, C. Walsh et al., "Propulsive power contribution of a kite and a Flettner rotor on selected shipping routes," Applied Energy, vol. 113, pp. 362-372, 2014.

[17] A. De Marco, S. Mancini, C. Pensa, R. Scognamiglio, and L. Vitiello, "Marine application of flettner rotors: numerical study on a systematic variation of geometric factor by DOE approach," in Proceedings of the 6th International Conference on
Computational Methods in Marine Engineering (MARINE '15), vol. 1, Rome, Italy, June 2015.

[18] CD-Adapco, Star-CCM+ User Guide, 2015.

[19] W. L. Oberkampf and F. G. Blottner, "Issues in computational fluid dynamics code verification and validation," AIAA Journal, vol. 36, no. 5, pp. 687-695, 1998.

[20] F. Stern, R. V. Wilson, H. W. Coleman, and E. G. Paterson, "Comprehensive approach to verification and validation of CFD simulations - part 1: methodology and procedures," Journal of Fluids Engineering, vol. 123, no. 4, pp. 793-802, 2001.

[21] P. J. Roache, Verification and Validation in Computational Science and Engineering, Hermosa, New Mexico, NM, USA, 1998.

[22] P. J. Roache, "Code verification by the method of manufactured solutions," Journal of Fluids Engineering, vol. 124, no. 1, pp. 4-10, 2002.

[23] I. B. Celik, U. Ghia, P. J. Roache, C. J. Freitas, H. Coleman, and P. E. Raad, "Procedure for estimation and reporting of uncertainty due to discretization in CFD applications," Journal of Fluids Engineering, vol. 130, no. 7, 2008.

[24] R. Cosner, W. L. Oberkampf, C. L. Rumsey, C. Rahaim, and T. Shih, "AIAA Committee on standards for computational fluid dynamics: status and plans," AIAA Paper 2006-889, American Institute of Aeronautics and Astronautics, Reno, Nev, USA, 2006.

[25] R. Wilson, J. Shao, and F. Stern, "Discussion: criticism of the correction factor," Journal of Fluids Engineering, vol. 126, no. 4, pp. 704-706, 2004.

[26] R. V. Wilson, F. Stern, H. W. Coleman, and E. G. Paterson, "Comprehensive approach to verification and validation of CFD simulations-part 2: application for RANS simulation of a cargo/container ship," Journal of Fluids Engineering, vol. 123, no. 4, pp. 803-810, 2001.

[27] T. Xing, P. Carrica, and F. Stern, "Computational towing tank procedures for single run curves of resistance and propulsion," Journal of Fluids Engineering, vol. 130, no. 10, Article ID 101102, 14 pages, 2008.

[28] F. Balsamo, F. De Luca, and C. Pensa, "A new logic for controllable pitch propeller management," in Proceedings of the 14th International Congress of the International Maritime Association of the Mediterranean (IMAM '11), vol. 2, pp. 639647, Genoa, Italy, September 2011. 


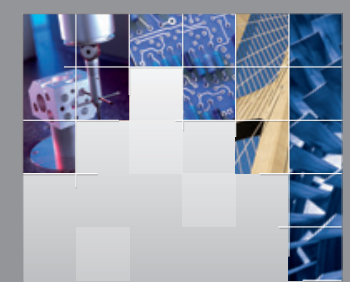

\section{Enfincering}
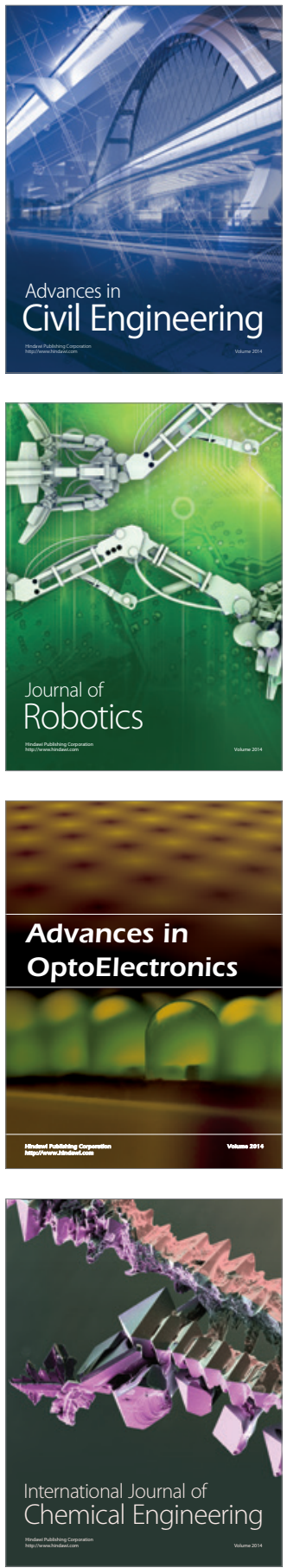

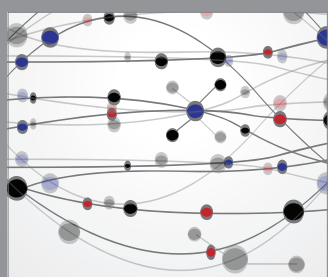

The Scientific World Journal

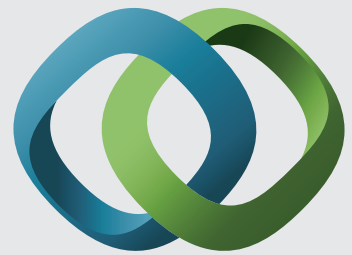

\section{Hindawi}

Submit your manuscripts at

http://www.hindawi.com
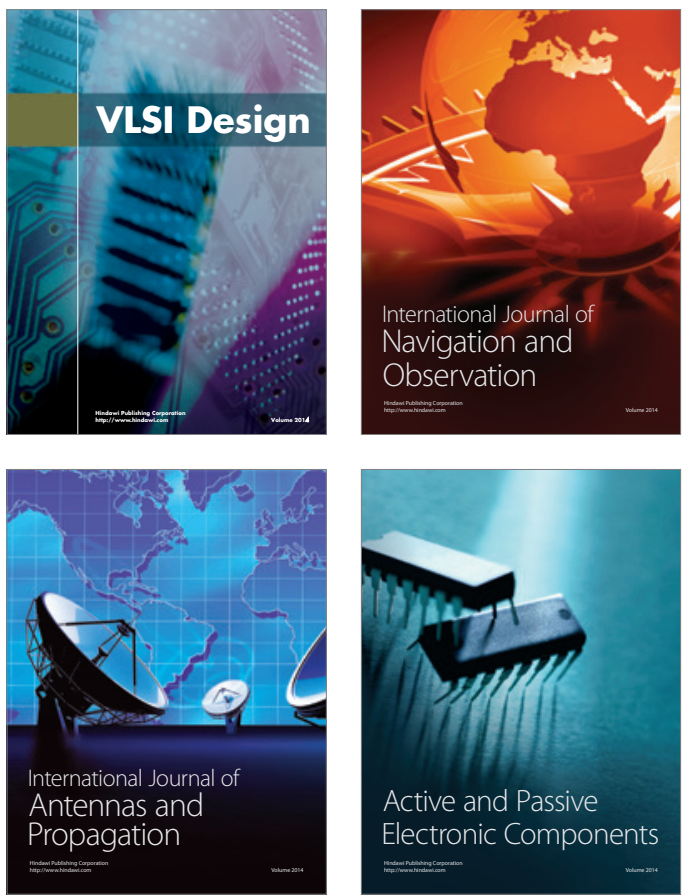
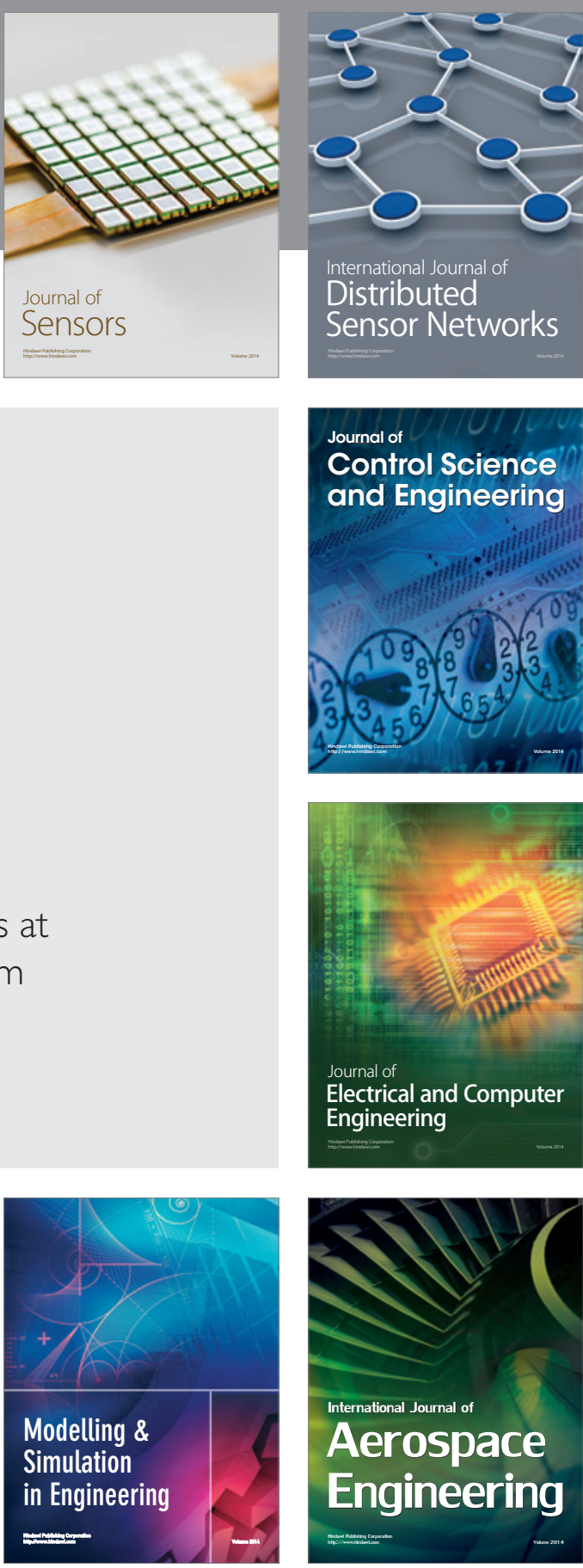

International Journal of

Distributed

Sensor Networks

Journal of

Control Science

and Engineering
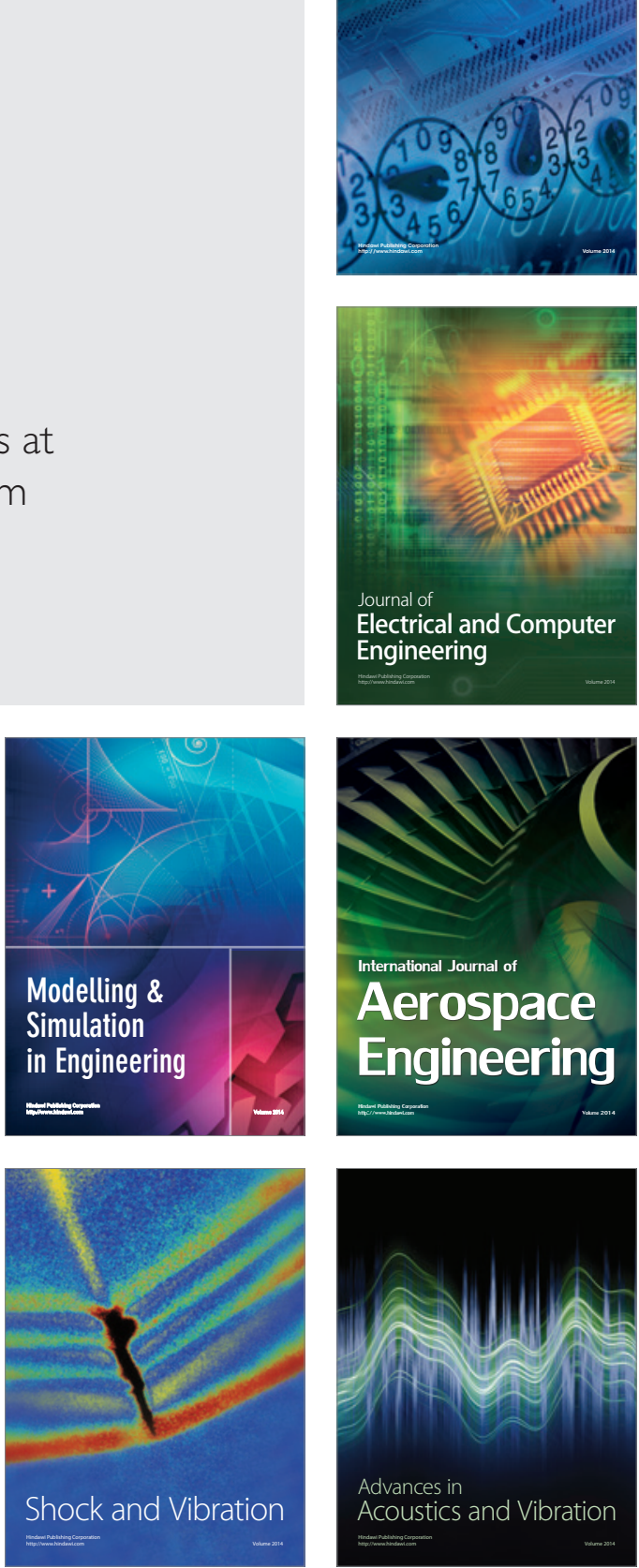